\title{
Improved performance of Xpert MTB/RIF assay on sputum sediment samples obtained from presumptive pulmonary tuberculosis cases at Kibong'oto infectious diseases hospital in Tanzania
}

Peter M. Mbelele ${ }^{1,2^{*}}$, Said Aboud ${ }^{2}$, Stellah G. Mpagama ${ }^{1}$ and Mecky I. Matee ${ }^{2}$

\begin{abstract}
Background: The introduction of Xpert MTB/RIF assay (Xpert) has significantly improved diagnosis of Tuberculosis (TB) in resource limited human immunodeficiency virus (HIV) endemic settings. We aimed to modify the Xpert protocol to improve the detection of Mycobacterium tuberculosis (MTB).

Methods: This cross sectional study was conducted among presumptive pulmonary tuberculosis (PTB) patients at Kibong'oto Infectious Diseases Hospital between August and November 2015. Each patient consented to provide 2 samples of raw sputa. One-sputum sample was sedimented using the Petroff's method and divided into two portions. One portion of sediment was inoculated on Lowenstein-Jensen culture media and observed for any growth for up to 8 weeks. Both, raw sputum and the portions of sediments were tested separately using Xpert with a sample reagent ratio of 1:2. Mean age of patients, prevalence of MTB, Xpert sensitivity, specificity, positive and negative predictive value were calculated. An incremental sensitivity was determined. Pearson chi-square and either an independent T or MannWhitney U-test were used to compared categorical and continuous variables respectively. A $p$ - value of $\leq 0.05$ was considered significant.
\end{abstract}

Results: Of the 270 presumptive PTB cases, 262 were eligible for analysis. Eight (3\%) were excluded due to contaminated culture. Patients' mean age was $42.9( \pm$ SD 15.1) years of which $173(66 \%)$ were female. The overall prevalence of PTB was 112 (43\%), of which the Xpert detected 105 (40\%) in sediments and 98 (37\%) in raw sputa as compared to culture which detected 85 (32\%) cases of PTB. Sensitivity, specificity, positive and negative predictive values of Xpert on sputum sediments were $92 \%, 85 \%, 74 \%$ and $96 \%$ respectively. Overall, the incremental sensitivity of Xpert on sediment over raw sputum was 6\%. In HIV infected Presumptive PTB, the incremental sensitivity was 12\%.

Conclusion: Lowering the sample reagent to sediment dilution ratio increases sensitivity of Xpert on MTB detection among presumptive PTB cases, especially in HIV infected individuals.

Keywords: Tuberculosis, Xpert ${ }^{\circledR}$ MTB/RIF, Sputum-sediments, Presumptive-tuberculosis, Kibong'oto, Tanzania

\footnotetext{
* Correspondence: mbelelepeter@yahoo.com

'Kibong'oto Infectious Diseases Hospital, P.O BOX 12 Sanya Juu, Kilimanjaro,

Tanzania

${ }^{2}$ Department of Microbiology and Immunology, School of Medicine,

Muhimbili University of Health and Allied Sciences, P.O BOX 65001 Dar es

Salaam, Tanzania
}

C) Bïmed Central (c) The Author(s). 2017 Open Access This article is distributed under the terms of the Creative Commons Attribution 4.0 International License (http://creativecommons.org/licenses/by/4.0/), which permits unrestricted use, distribution, and reproduction in any medium, provided you give appropriate credit to the original author(s) and the source, provide a link to the Creative Commons license, and indicate if changes were made. The Creative Commons Public Domain Dedication waiver (http://creativecommons.org/publicdomain/zero/1.0/) applies to the data made available in this article, unless otherwise stated. 


\section{Background}

Tanzania remains one of the countries with a high burden of tuberculosis (TB) and human immunodeficiency virus (HIV) co-infection [1]. Despite the consolidated control strategies, the incidence of TB and mortality rate in both $\mathrm{TB}$ and $\mathrm{TB}$ with HIV co-infected cases has remained steady [2]. A key challenge in TB/HIV control includes the difficulty in correct diagnosis due to alteration of clinical presentation resulting in a delay of appropriate treatment [3]. Important factors that delay early diagnosis of TB include the inability of TB/HIV coinfected patients to produce quality sputum for diagnosis [4]. Previously studied methods for improving the quality of sputum for TB diagnosis include overnight pooling [5] and processing sputum with different chemicals such as N-Acetyl-L-Cysteine (NALC) to concentrate Mycobacterium tuberculosis (MTB) [6]. Recent technologies to increase the sensitivity of MTB detection in samples include light emitted diode microscopy [7], and rapid molecular diagnostics such as Xpert [8].

The introduction of Xpert (Cepheid, Sunnyvale USA) in particular has been a major breakthrough in TB diagnostics, especially in resource limited HIV endemic settings. The assay is a semi-automated real time Polymerase Chain Reaction (PCR) that was approved by the World Health Organization (WHO) in 2010 for dual detection of MTB and rifampicin resistance [8]. A systematic review of Xpert has shown an excellent performance compared to conventional smear microscopy for acid fast bacilli (AFB) and Liquid or Lowenstein Jensen (LJ) solid culture methods. Compared with smear microscopy, a point of care test for TB diagnosis in most resource limited settings, Xpert considerably increases TB detection among culture-confirmed cases accounting for pooled sensitivity and specificity of 89\% and 99\% respectively [9]. However, Xpert has higher sensitivity in detecting MTB in samples that were collected from patients with smear-positive results for AFB than smear-negative [10]. Smear negativity is a common phenomenon among HIV positive patients and the performance of Xpert on pooled samples of HIV individuals was $79 \%$ and $86 \%$ sensitivity and specificity respectively [9]. However, like other molecular diagnostics, Xpert suffers a diminished effect in the HIV population [11].

The commonly applied protocol for detecting MTB in Xpert at our hospital and in most other settings is the use of raw sputum to Xpert sample reagent (SR) with a ratio of $1: 2[10,12]$. The SR contains $2 \%$ of sodium hydroxide $(\mathrm{NaOH})$ and isopropanol. However, manufacturer of Xpert recommends the use of either raw, unprocessed sputa or concentrated sputum sediments. Here, the raw sputum sample is liquefied, decontaminated either with $2 \% \mathrm{~N}$ acetyl cysteine-sodium hydroxide (NALC-NaOH) or $4 \%$ $\mathrm{NaoH}$, centrifuged, concentrated and neutralized using phosphate buffer [13]. These sediments have higher load of MTB compared to raw sputum [14]. Nevertheless, previous reports for MTB detection in Xpert using processed sputum sediments did not show any difference to raw sputum $[10,14]$. These studies reportedly used a raw and processed sputum to SR ratio of $1: 2$ and 1:3 respectively [15]. Using sputum sediments from patients with TB/HIV co-infection, Dharan et al. [16] found high detection rate of MTB when the sediment/SR ratio was changed to 1:2. Our hypothesis is that decreasing the Xpert SR dilution will increase the probability of detecting MTB among PTB suspects in resource limited HIV endemic settings like ours. While the raw sputum was processed as per manufacturer's protocol, we sought to modify the Xpert protocol by particularly adjusting the dilution of sediment to SR with a ratio of 1:2 instead of 1:3 as recommended. Using LJ culture medium as a reference method, we determined the incremental detection value of MTB on sputum sediment samples.

\section{Methods \\ Study design and setting}

This cross sectional study design was conducted at Kibong'oto Infectious Diseases Hospital (KIDH) located in Siha District, Kilimanjaro region, Tanzania. KIDH is a public hospital with bed capacity of 320 . It is the National Centre of excellence for clinical management of drug resistant TB in the country. The hospital provides TB services to more than 150 and 500 patients with drug resistant and susceptible TB per year respectively. KIDH has a public health laboratory supported by East African Public Health Laboratory Networks through the World Bank Scheme. Since 2016, the laboratory has established drug resistance TB surveillance system and is now in the process of accreditation. The laboratory has facilities for mycobacterial culture on LJ solid media, smear microscopy for AFB (LED microscopy), Xpert (Cepheid, USA) and Line Probe assay (GenoTypeMTBDR $_{\text {plus }}$ that detects MTB; and isoniazid and rifampicin susceptibility and GenoTypeMTBDR sl $_{\text {for }}$ detecting MTB and their susceptibility to second line injectable drugs and flouroquinolones). The average number of sputum samples processed per day for culture and molecular testing ranges from 25 to 30 samples.

\section{Recruitment and evaluation of study participants}

Participants were presumptive pulmonary TB cases aged $\geq 18$ years and were enrolled after obtaining a written informed consent. A standardized semi-structured questionnaire containing a set of study variables was used to collect data from study participants and medical charts. Data collected included symptoms and signs suggestive of PTB, any previous history of TB treatment, HIV status, absolute CD4 + T cell count and socio-demographic characteristics such as age, gender, occupation etc. 


\section{Sample size calculations}

A minimum sample size of 256 was determined using Buderer's formula for diagnostic tests. A prevalence of TB among TB suspect cases of 33.5\% reported by Meremo et al. [17] and anticipated average Xpert sensitivity for raw sputum and sediment of $95 \%$ at a significance level of 5\% was used [14]. Because of an anticipated culture contamination rate of about $5 \%$ on LJ media, 14 participants were added to the sample size. Therefore a total of 270 participants were enrolled.

\section{Study procedures}

\section{Samples collection and processing}

Each study participant provided two spot sputum samples $30 \mathrm{~min}$ apart. One of the two samples was processed using the modified Petroff's method [18]. Briefly, $3 \mathrm{mls}$ of sputum was added to $3 \mathrm{mls}$ of $4 \%$ sodium hydroxide $(\mathrm{NaOH})$. The mixture was vortexed and left to stand at room temperature for $15 \mathrm{~min}$. Thereafter, sterile distilled water was added to a 50mark of falcon tube and concentrated by centrifugation at $3000 \mathrm{~g}$ for $15 \mathrm{~min}$. Supernatants were discarded into a container with 25\% phenol. Sediments were suspended in Phosphate buffer solution (PBS) before being split into 2 portions, one for testing with the Xpert and the other for the LJ culture media as recommended by the Clinical and Laboratory Standard Institute (CLSI) for TB culture [13]. The second unprocessed raw sputum was for direct testing using the Xpert.

\section{Detection of MTB in raw sputum and sediments collected from presumptive PTB patients by Xpert}

The raw sputum was tested as recommended by the manufacturer of Xpert, here referred to as standard dilution. Unprocessed raw sputum was diluted with sample reagent (SR) at a ratio of 1:2 [15]. Sputum sediments were tested using a modified protocol, referred to as experiment dilution. In this protocol, sediments were diluted with SR at a ratio of 1:2. Both, raw and sediment samples were incubated at room temperature for $15 \mathrm{~min}$ to reduce MTB viability by $10^{6}$ fold as recommended by the manufacturer. At least $2 \mathrm{ml}$ of either raw or sediments sample was transferred into the cartridge and loaded into the Xpert module to continue with automatic DNA extraction, amplification and detection of MTB. The MTB detection is done by amplifying MTB specific sequence of the $r p o B$ gene probed with five molecular beacons A, B, C, D and E, each labeled with a unique fluorophore. During detection, the valid maximum cycle threshold (CT) was 39.0 for Probes A, B and $\mathrm{C}$ and 36.0 for Probes D and E. The MTB is detected when at least two probes result in $\mathrm{CT}$ values within the valid range and a delta $\mathrm{CT}$ min of less than 2.0. Depending on the CT value, the MTB is semi-quantified into very low, low, medium and high for CT values of $>28$, 23-28, 16-22 and <16 cycles respectively [19]. Similarly, the assay does not detect MTB when there is only one or no positive probe.

\section{Culture of MTB on Lowenstein Jensen (LJ) medium}

Part of the sputum sediment was cultured on LJ solid medium, a reference method, as per CLSI [13]. In summary, $200 \mu \mathrm{l}$ of sputum sediments were inoculated on two slopes of LJ medium containing either pyruvate or glycerol. For each batch of the sputum sample cultured, a standard laboratory strain MTB H37Rv strain and uninoculated LJ medium was used as positive and negative quality control of culture, respectively. Inoculated LJ media were incubated at $37{ }^{\circ} \mathrm{C}$ and observed weekly for up to 8 weeks before declared negative. MTB colonies were identified and reported according to locally existing and CLSI standard operating procedures.

\section{Data management and statistical analysis}

The semi-structured questionnaire was used as a gold standard tool for data collection from participants and medical charts. Raw data collected were verified for correctness before being entered into EpiData software version 3.1. Data on EpiData were cleaned before analysis using Statistical Package for Social Sciences version 23.0. They were summarized in proportion with percentages or estimated with measure of central tendency with standard deviation of the mean or 95\% confidence interval accordingly. Sensitivity, specificity, positive and negative predictive values with $95 \% \mathrm{CI}$ of Xpert on raw sputum and sediments was calculated using diagnostic test evaluation calculator- MedCalc Statistical Software version 16.4.3 (MedCals software bvba, Ostend, Belgium; https://www.medcalc.org; 2016) [20]. Incremental value of Xpert on raw and sputum sediment was also calculated using the LJ culture as a reference method. The Pearson Chi-square was calculated for categorical variables such as gender, occupation, HIV status and presenting symptoms. Independent T-test and MannWhitney $U$ test were used to compare PTB cases with continuous variables like age and absolute CD4+ T cell count respectively. A two-tailed test statistic was considered significant for a $p$-value of $\leq 0.05$.

\section{Results}

\section{Characteristics of study participants}

From August through November 2015, 270 presumptive PTB participants consented and provided 2 samples of raw sputa making a total of 540 sputa. However, 16 sputa samples that were collected from 8 participants were not analyzed due to culture contamination (Fig. 1). Therefore, 262 raw sputa were tested on Xpert and the other 262 decontaminated sputa were centrifuged to 


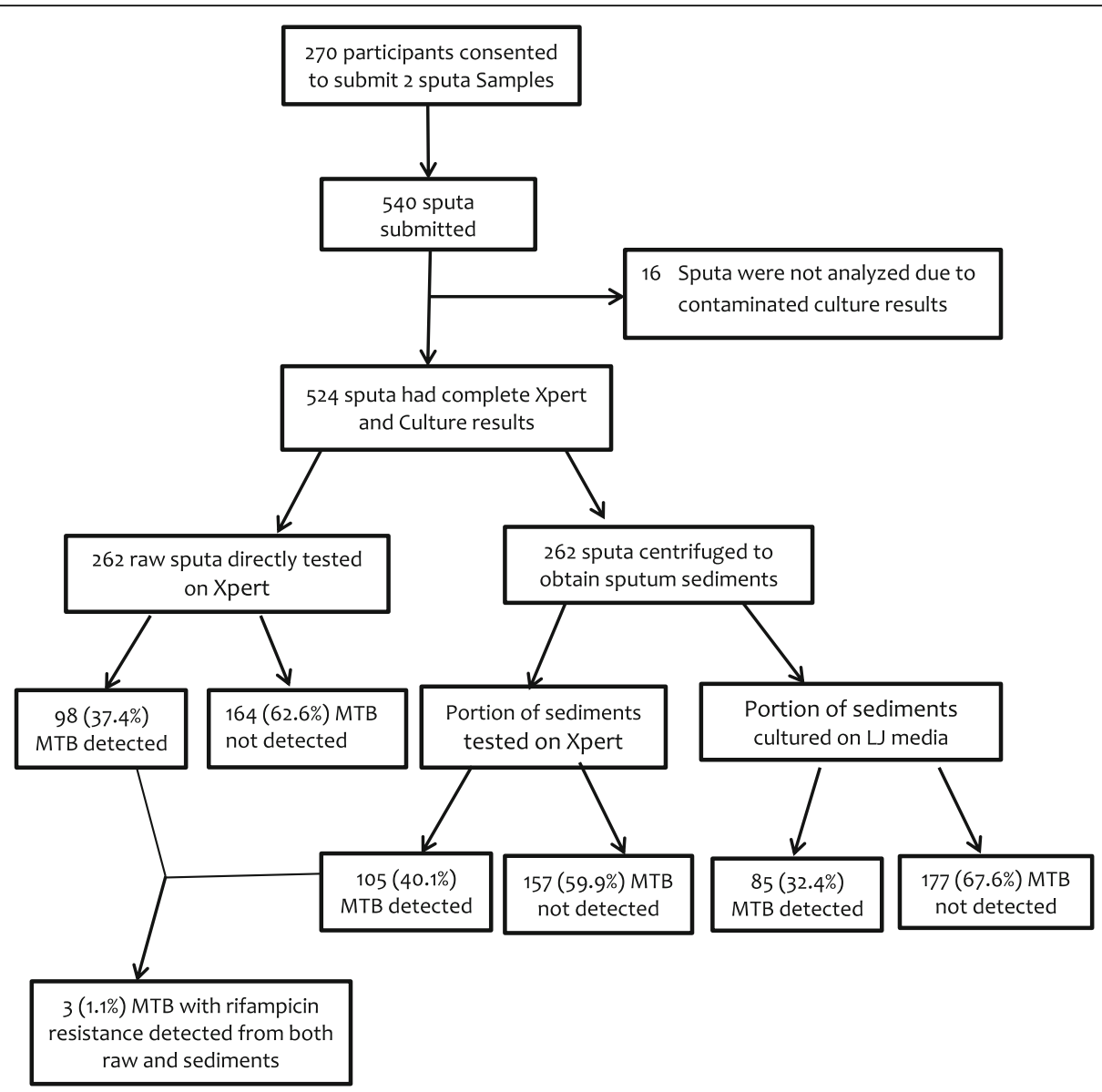

Fig. 1 Study Procedures and proportion of PTB

obtain sputum sediments. Of the 262 participants; 173 (66\%) were female. Their mean age was $42.9( \pm$ SD 15.1) years (Table 1$)$. Thirty nine (14.9\%) and $36(13.7 \%)$ of them had HIV infection and prior exposure to anti-TB treatment respectively (Table 1 ). The median absolute CD4 + T cell counts of HIV infected participants was 285 (IQR: 162-423) cells/ $\mu \mathrm{L}$. The main clinical presenting symptoms included cough in 262 cases (100\%), hemoptysis in 41 (16\%), chest pain/difficulty breathing in 240 (92\%) cases, fever in 241 (92\%), excessive night sweat in 172 (66\%) and weight loss in $203(78 \%)$ cases.

\section{Performance characteristics of Xpert and incremental sensitivity on raw and sediment samples}

Almost all samples that were collected from study participants had valid Xpert results. These results were similar to those obtained from the standard MTB H37Rv strain and de-ionized water for positive and negative quality control samples respectively. However, four raw sputum samples showed no Xpert results due to prolonged interruption of the laboratory's power supply. The results were valid when Xpert was repeated. Similarly, one sediment sample had MTB with indeterminate rifampicin resistance (RR). This sample had MTB without RR when Xpert was repeated. The prevalence of PTB detected either by Xpert on raw sputa and sediments or LJ culture method that was collected from presumptive PTB patients was 112 (43\%). The Xpert detected 105 (40.1\%) on sputum sediments while detection on raw and culture were 98 (37.4\%) and $85(32.4 \%)$ respectively (Fig. 1). However, the Xpert detected $3(1.1 \%)$ cases with rifampicin resistance from both raw and sediments (Fig. 1). Of PTB cases detected on sediments, the Xpert semi-quantified MTB as very low 6 (6\%), low 27 (26\%), moderate 37 (35\%) and high 35 (33\%).The overall sensitivity of Xpert on sputum sediments was higher than that on raw sputa. Notably, sensitivity in HIV infected presumptive PTB cases was excellent (Table 2). Incremental sensitivity value for all study participants was $6 \%$, while for the HIV infected PTB cases it was $12 \%$. Also, the Xpert detected MTB in 27 (10\%) of presumptive PTB cases that were negative on LJ culture, a gold standard. Further examination of these cases revealed that only 3 (11\%) had a history of previous 
Table 1 Baseline characteristics of study participants $(N=262)$

\begin{tabular}{lll}
\hline Characteristics & Frequency & $\%$ \\
\hline Age group (years) & & \\
$\leq 24$ & 28 & 10.7 \\
$25-34$ & 46 & 17.6 \\
$35-44$ & 87 & 33.2 \\
$45-54$ & 47 & 17.9 \\
$55+$ & 54 & 20.6 \\
Sex & & \\
Male & 89 & 34 \\
Female & 173 & 66 \\
Occupation & & \\
Employed & 8 & 3.1 \\
Small business & 60 & 22.9 \\
Peasant & 168 & 64.1 \\
Mining casual workers & 26 & 9.9 \\
HIV status & & \\
Positive & 39 & 14.9 \\
Negative & 223 & 85.1 \\
Median CD4+ T cells counts; $\mathrm{n}$ (IQR) cells/ $\mathrm{LL}$ & 285 (162-423) \\
History of prior TB treatment & & \\
Yes & 36.7 \\
No & 226 & \\
\hline
\end{tabular}

Table 2 Performance Characteristics of Xpert on sediments and raw sputum samples

Performance characteristics of Xpert in Presumptive PTB using $L$ culture as a reference method $(N=262)$

Sensitivity (\%) Specificity (\%) PPV (\%) NPV (\%) P-value

Performance characteristics of Xpert on sputum sediments using experimental dilution (1:2)

92 (84-97) $\quad 85$ (79-90) $\quad 74(67-80) \quad 96(91-98) \quad 0.0001$

Performance characteristics of Xpert on raw sputa using standard dilution (1:2)

$86(77-93) \quad 86(77-93) \quad 75(65-83) \quad 93(88-96) \quad 0.001$

Incremental values

$\begin{array}{llll}6 & 1 & 1 & 3\end{array}$

Performance characteristics of Xpert in HIV Infected presumptive PTB $(n=39)$

Performance characteristics of Xpert on sputum sediments using experimental dilution (1:2)

$$
100(81-100) \quad 82(60-95) \quad 81(64-91) \quad 100 \quad 0.0001
$$

Performance characteristics of Xpert on raw sputa using standard dilution (1:2)

$88(64-99) \quad 86(65-97) \quad 83(59-96) \quad 91(70-99) \quad 0.0001$

Incremental values

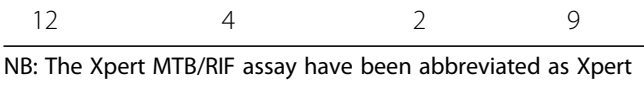

PTB treatment. Likewise, culture detected 7 (3\%) presumptive PTB cases that were missed by Xpert.

\section{Discussion}

The main finding of this study is that sputa sediments to Xpert SR ratio of 1:2 considerably increase the sensitivity of detecting MTB, especially in HIV infected presumptive PTB cases. Our findings are in agreement with those of Dharan et al. [16] who had a high detection rate of MTB when the sediment/SR ratio was lowered to 1:2. These findings support the use of sputum sediments on Xpert especially in a TB and HIV co-infection endemic setting such as Tanzania and across Sub-Saharan Africa. Although this procedure presents additional costs of about 5-10 USD per sample [21] and time for centrifuging the raw sputa, the cost incurred for treating a patient inadequately, especially if cases are missed, outweighs the cost for the modified protocol. Ineffective diagnostics prompts multiple hospital visits causing additional costs for consultation, transportation to health facility and repeated Xpert test in a heavily subsidized health system. For example, in low and middle income countries like Tanzania, the cost of treating a patient with drug susceptible TB ranges from 250-300USD [22].

In the present study, the specificity of Xpert on sputa sediments among HIV infected participants was $4 \%$ lower compared to that in raw sputa, as one more nonPTB HIV case was falsely determined as positive on sediments (Table2). Remarkably, the Xpert detected 26\% more MTB cases on sputum sediment than LJ culture (Table2). Certainly, the difference could be attributable to inability of Xpert to discriminate between viable and non-viable MTB DNA. This is a common phenomenon in previously treated, PTB cases, resulting in false positives $[23,24]$. However, only $11 \%$ of the false positive population had a history of previous PTB treatment. This finding, which is in keeping with those by Geleta DA et al. [25], could partly result from participants deliberately giving false TB treatment history [26]. On the other hand, Xpert sediment results had an average of $4 \%$ false negatives (Table 2). Indeed, this might be due to a difference in detection limit of Xpert, which requires higher number of AFB than culture methods. For example, the estimated detection limit of Xpert is 131 colonies forming unit $(\mathrm{CFU}) / \mathrm{ml}$, while LJ culture is 10 $100 \mathrm{CFU} / \mathrm{ml}$ [27].

The notable strength of this study is the detection of 7 PTB cases more on the modified protocol for sediments that were missed on raw sputa (Fig. 1). In addition, the Xpert detected 3 out of 262 (1.1\%) cases with rifampicin resistance tuberculosis (RR-TB) in both, raw sputa and sediments that were collected from patients. These cases received treatment for multi-drug resistance tuberculosis (MDR-TB) as per existing guideline in Tanzania and 
elsewhere [28, 29]. This low proportion of RR-TB best align with findings by Nagu et al. who found uncommon cases of MDR-TB among patients without prior exposure to anti-TB drugs in Tanzania [30]. Despite these strengths, our study has some limitations. We were unable to compare the performance characteristics of Xpert with smear microscopy results because KIDH has shifted the practice of using smear microscopy as the frontline TB diagnostic to Xpert as recommended by the WHO. Therefore, we were unable to correlate Xpert performance characteristics with sputum AFB density. However, the CT values have not been shown to correlate with smear for AFB density [31] and hence cannot affect validity of the current findings. Also, our results did not compare the ratio of sputum sediment to Xpert SR of 1:3 to that of modified protocol (1:2) and we recommend further study to decipher any difference in detection of MTB.

\section{Conclusion}

Lowering sediment to SR dilution ratio to 1:2 improved performances of Xpert on Sputum Sediment Samples, especially those obtained from HIV-infected individuals. However, operational and cost-effective studies will be required to determine the feasibility of the national $\mathrm{TB}$ program in implementing the proposed modified protocol sustainably.

\section{Abbreviations}

AFB: Acid fast bacilli; AIDS: Acquired Immunodeficiency syndrome; CFU: Colonies forming unit; CLSI: Clinical and Laboratory Standard Institute; CT: Cycle threshold; HIV: Human Immunodeficiency Virus; KIDH: Kibong'oto Infectious Diseases Hospital; LJ: Lowenstein Jensen; MDR-TB: Multidrug resistant Tuberculosis; MTB: Mycobacterium tuberculosis; NALC-NaOH: NAcetyl-L-Cysteine Sodium Hydroxide; NPV: Negative predictive value; PPV: Positive Predictive value; PTB: Pulmonary tuberculosis; RR: Rifampicin resistance; SR: Sample reagent; TB: Tuberculosis; WHO: World Health Organization; Xpert: Xpert MTB/RIF assay

\section{Acknowledgements \\ The authors would like to thank KIDH Hospital Acting Medical Office Incharge, Dr. Riziki Kisonga, (M.D) for giving permission to conduct this study. We also thank Ms. Mary Mtui, a laboratory quality officer at KIDH for ensuring all laboratory works are done in compliance with ISO: 15189.2012.The author would also thank Ms. Batuli Mono and Taji Mnzava who worked with authors during data collection. Lastly, the Authors extend their appreciation to Dr. Katharina Kreppel (PhD), a native English speaker from the University of Glasgow for her intensive review and correction of English language in the manuscript.}

\section{Funding}

The study was funded by the Ministry of Health, Community Development, Gender, Elderly and Children of Tanzania through World Bank, East Africa Public Health Laboratory Networking Project. However, the funding agent had no influence in the design of the study, collection, analysis, and interpretation of data and in writing the manuscript.

\section{Availability of data and materials}

The datasets used and/or analyzed during the current study are available from the corresponding author on reasonable request.

\section{Authors' contributions}

PMM: conceived, designed the study, collected data, performed statistical analysis, interpretation of results and wrote the manuscript. SGM: performed statistical analysis, interpretation of results and wrote the manuscript, MIM: Made interpretation of results, thoroughly reviewed and wrote the manuscript. SA: supervised the whole process from study design, data collection, and performed interpretation of results and execution of manuscript writing. All authors read and approved the final version of the manuscript.

\section{Ethics approval and consent to participate}

Ethical approval was obtained from Muhimbili University of Health and Allied Sciences Senate Research and Publications Committee, whose reference number was Ref.No.MU/PGS/SAECNol.XVI/. Permission to conduct the study was obtained from $\mathrm{KIDH}$ administration. Prior to any study procedure, we obtained a written informed consent from participants. All study participants with PTB received anti-tuberculosis treatment according to existing National TB treatment guidelines in Tanzania [1].

\section{Consent for publication}

Not applicable.

\section{Competing interests}

The authors declare that they have no competing interests.

\section{Publisher's Note}

Springer Nature remains neutral with regard to jurisdictional claims in published maps and institutional affiliations.

Received: 28 July 2017 Accepted: 19 December 2017

Published online: 29 December 2017

\section{References}

1. National Tuberculosis and Leprosy Programme (NTLP). United Republic of Tanzania Ministry of Health and Social Welfare. Manual for the Management of Tuberculosis and Leprosy, Sixth Edition. 2013.

2. World Health Organisation (WHO). Global tuberculosis report 2016. World Health Organisation. 20 Avenue Appia, 1211 Geneva 27, Switzerland; 2016.

3. Raviglione M, Sulis G. Tuberculosis 2015: burden, challenges and strategy for control and elimination. Infect Dis Rep. 2015;8(2):33-7.

4. Kibiki GS, Mulder B, Van Der Ven AJAM, Sam N, Boeree MJ, Van Der Zanden A, Dolmans WMV. Laboratory diagnosis of pulmonary tuberculosis in TB and HIV endemic settings and the contribution of real time PCR for M. Tuberculosis in bronchoalveolar lavage fluid. Trop Med Int Heal. 2007;12(10):1210-7.

5. Mpagama SG, Mtabho C, Mwaigwisya S, Mleoh L, Boer IMS, Heysell SK, Houpt ER, Kibiki GS. Comparison of overnight pooled and standard sputum collection method for patients with suspected pulmonary tuberculosis in northern Tanzania. Tuberc Res Treat. 2012;2012:1-5.

6. Steingart KR, Ng V, Henry M, Hopewell PC, Ramsay A, Cunningham J, Urbanczik R, Perkins MD, Aziz MA, Pai M. Sputum processing methods to improve the sensitivity of smear microscopy for tuberculosis: a systematic review. Lancet Infect Dis. 2006:6(10):664-74.

7. World Health Organisation (WHO). Same-day diagnosis of tuberculosis by microscopy: policy statement. World Heal Organ Doc. 2009:47(1):8-14.

8. World Health Organisation (WHO). Rapid implementation of the Xpert MTB/ RIF diagnostic test. Technical and operational "How to" practical considerations. World Health Organisation Doc. 2011:WHO/HTM/TB:1-34.

9. Steingart K, Schiller I, Dj H, Pai M, Cc B, Dendukuri N, Steingart KR, Schiller I, Horne DJ, Pai M, Boehme CC, Dendukuri N. Xpert ${ }^{\circledR}$ MTB / RIF assay for pulmonary tuberculosis and rifampicin resistance in adults. Cochrane Libr. 2014;1:1-132.

10. Boehme CC, Nicol MP, Nabeta P, Michael JS, Gotuzzo E, Tahirli R, Gler MT, Blakemore R, Worodria W, Gray C, Huang L, Caceres T, Mehdiyev R, Raymond L, Whitelaw A, Sagadevan K, Alexander H, Albert H, Cobelens F, Cox H, Alland D, Perkins MD. Feasibility, diagnostic accuracy, and effectiveness of decentralised use of the Xpert MTB/RIF test for diagnosis of tuberculosis and multidrug resistance: a multicentre implementation study. Lancet. 2011 Apr 30;377(9776): 1495-505.

11. Theron G, Zijenah L, Chanda D, Clowes P, Rachow A, Lesosky M, Bara W, Mungofa S, Pai M, Hoelscher M, Dowdy D, Pym A, Mwaba P, Mason P, Peter J, Dheda K. Feasibility, accuracy, and clinical effect of point-of-care Xpert MTB/RIF testing for tuberculosis in primary-care settings in Africa: a multicentre, randomised, controlled trial. Lancet. 2014 Feb 1;383(9915):424-35. 
12. Mollel EW, Chilongola JO, Mpagama SG, Kibiki GS. Evaluation of XpertMTB/ Rif performance for diagnosis of tuberculosis among HIV positive patients in northern Tanzania. Tanzan J Health Res. 2017;19(1):1-9.

13. Clinical and Laboratory Standards Institute (CLSI). Laboratory detection and identification of mycobacteria; approved standard. CLSI document M48-A. 2008.

14. Darwish M, Abd M, Wadood E, Alnagdi H. Diagnostic assessment of Xpert MTB / RIF in a sample of mycobacterium tuberculosis Egyptian patients. African J. Microbiol Res. 2013;7(44):5107-13.

15. Cepheid ${ }^{\oplus}$. Xpert MTB / RIF Package Insert. 2009. http://tbevidence.org/ documents/rescentre/sop/XpertMTB Broch_R9_EU.pdf

16. Dharan NJ, Amisano D, Mboowa G, Ssengooba W, Blakemore R, Kubiak RW, Armstrong DT, Jones M, Manabe YC, Joloba ML, Ellner JJ, Dorman SE, Alland D. Improving the sensitivity of the Xpert MTB/RIF assay on sputum pellets by decreasing the amount of added sample reagent: a laboratory and clinical evaluation. J Clin Microbiol. 2015;53(4):1258-63.

17. Meremo AJ, Kidenya BR, Mshana SE, Kabangila R, Kataraihya JB. High prevalence of tuberculosis among adults with fever admitted at a tertiary hospital in north-western Tanzania. Tanzan J Health Res. 2012;14(3):1-9.

18. Tripathi K, Tripathi PC, Nema S, Shrivastava AK, Dwiwedi K. Modified Petroff ' $s$ Method : an Excellent Simplified Decontamination Technique in Comparison with Petroff' s Method 2014;10(3):461-4.

19. Ssengooba W, Respeito D, Mambuque E, Blanco S, Bulo H, Mandomando I, De JBC, Cobelens FG. Do Xpert MTB / RIF cycle threshold values provide information about patient delays for tuberculosis diagnosis? PLoS One. 2016;302:1-10

20. MedCalc Statistical Software version 16.4.3 (MedCals software bvba, Ostend, Belgium; 2016. https://www.medcalc.org.

21. Shah M, Chihota V, Coetzee GJ, Churchyard GJ, Dorman SE. Comparison of laboratory costs of rapid molecular tests and conventional diagnostics for detection of tuberculosis and drug-resistant tuberculosis in South Africa. BMC Infect Dis. 2013;13(1):352.

22. Laurence YV, Griffiths UK, Vassall A, Laurence W. Costs to health services and the patient of treating tuberculosis : a systematic literature review. PharmacoEconomics. 2015;33(9):939-55.

23. Chang K, Lu W, Wang J, Zhang K, Jia S, Li F, Deng S, Chen M. Rapid and effective diagnosis of tuberculosis and rifampicin resistance with Xpert MTB/ RIF assay: a meta-analysis. J Inf Secur. 2012 Jun;64(6):580-8.

24. Theron G, Venter R, Calligaro G, Smith L, Limberis J, Meldau R, Chanda D, Esmail A, Peter J, Dheda K. Xpert MTB/RIF Results in Patients With previous tuberculosis: can we distinguish true from false positive results? Clin Infect Dis 2016;62(8):995-1001.

25. Geleta DA, Megerssa YC, Gudeta AN, Akalu GT, Debele MT, Tulu KD, Xpert MTB. RIF assay for diagnosis of pulmonary tuberculosis in sputum specimens in remote health care facility. BMC Microbiol. 2015;15(1):220.

26. Mpagama Stellah, Chongolo Anna, Lyimo Jonson, Kisonga Riziki, Heysell Scott ME. Gridlock from Diagnosis to Treatment of Multidrug Resistant Tuberculosis (MDR-TB) in Tanzania: Patient Perspectives from Focus Group Discussions. In: 48th World Conference on Lung Health of the International Union Against Tuberculosis and Lung Disease (The Union). Guadalajara Mexico; 2017.

27. Helb D, Jones M, Story E, Boehme C, Wallace E, Ho K, Kop J, Owens MR, Rodgers R, Banada P, Safi H, Blakemore R, Ngoc Lan NT, Jones-López EC, Levi M, Burday M, Ayakaka I, Mugerwa RD, McMillan B, Winn-Deen E, Christel L, Dailey P, Perkins MD, Persing DH, Alland D. Rapid detection of mycobacterium tuberculosis and rifampin resistance by use of on-demand near-patient technology. J Clin Microbiol. 2010;48(1):229-37.

28. National Tuberculosis and Leprosy Programme (NTLP). United Republic of Tanzania. Ministry of Health and social welfare. Operational Guidelines for the Management of Drug Resistant TB in Tanzania, First Edition. 2012.

29. World Health Organisation (WHO). Companion handbook to the WHO guidelines for the programmatic management of drug-resistant tuberculosis. 2014

30. Nagu TJ, Aboud S, Mwiru R, Matee M, Fawzi W, Mugusi F. Multi drug and other forms of drug resistant tuberculosis are uncommon among treatment naïve tuberculosis patients in Tanzania. PLoS One. 2015;10(4):1-10.

31. Theron G, Pinto L, Peter J, Mishra HK, Mishra HK, Van Zyl-smit R, Sharma SK, Dheda K. The use of an automated quantitative polymerase chain reaction (Xpert MTB/RIF) to predict the sputum smear status of tuberculosis patients. Clin Infect Dis. 2012;54(3):384-8.

\section{Submit your next manuscript to BioMed Central and we will help you at every step:}

- We accept pre-submission inquiries

- Our selector tool helps you to find the most relevant journal

- We provide round the clock customer support

- Convenient online submission

- Thorough peer review

- Inclusion in PubMed and all major indexing services

- Maximum visibility for your research

Submit your manuscript at www.biomedcentral.com/submit
Biomed Central 